\title{
Islet Amyloid Polypeptide Inserts into Phospholipid Monolayers as Monomer
}

\author{
Maarten F. M. Engel ${ }^{1,2 *}$, HaciAli Yigittop ${ }^{2}$, Ronald C. Elgersma ${ }^{3}$ \\ Dirk T. S. Rijkers ${ }^{3}$, Rob M. J. Liskamp ${ }^{3}$, Ben de Kruijff ${ }^{2}$ \\ Jo W. M. Höppener ${ }^{1}$ and J. Antoinette Killian ${ }^{2}$
}

${ }^{1}$ Department of Metabolic and Endocrine Diseases, Division of Biomedical Genetics, University Medical Center Utrecht, $P O$

Box 85090, NL-3508 AB

Utrecht, The Netherlands

${ }^{2}$ Department of Biochemistry of Membranes, Centre of Biomembranes and Lipid Enzymology, Institute of Biomembranes, Utrecht University, Padualaan 8 NL-3584 CH Utrecht, The Netherlands

${ }^{3}$ Department of Medicinal Chemistry, Utrecht Institute for Pharmaceutical Sciences

Utrecht University,

Sorbonnelaan 16, NL-3584 CA

Utrecht, The Netherlands
Amyloid deposits in the pancreatic islets of Langerhans are thought to be a main factor responsible for death of the insulin-producing islet $\beta$-cells in type 2 diabetes. It is hypothesized that $\beta$-cell death is related to interaction of the 37 amino acid residue human islet amyloid polypeptide (hIAPP), the major constituent of islet amyloid, with cellular membranes. However, the mechanism of hIAPP-membrane interactions is largely unknown. Here, we study the nature and the molecular details of the initial step of hIAPPmembrane interactions by using the monolayer technique. It is shown that both freshly dissolved hIAPP and the non-amyloidogenic mouse IAPP (mIAPP) have a pronounced ability to insert into phospholipid monolayers, even at lipid packing conditions that exceed the conditions that occur in biological membranes. In contrast, the fibrillar form of hIAPP has lost the ability to insert. These results, combined with the observations that both the insertion kinetics and the dependence of insertion on the initial surface pressure are similar for freshly dissolved hIAPP and mIAPP, indicate that hIAPP inserts into phospholipid monolayers most likely as a monomer. In addition, our results suggest that the N-terminal part of hIAPP, which is nearly identical with that of mIAPP, is largely responsible for insertion. This is supported by experiments with hIAPP fragments, which show that a peptide consisting of the $19 \mathrm{~N}$-terminal residues of hIAPP efficiently inserts into phospholipid monolayers, whereas an amyloidogenic decapeptide, consisting of residues 20-29 of hIAPP, inserts much less efficiently. The results obtained here suggest that hIAPP monomers might insert with high efficiency in biological membranes in vivo. This process could play an important role as a first step in hIAPP-induced membrane damage in type 2 diabetes.

(C) 2005 Elsevier Ltd. All rights reserved.

Keywords: amylin; monolayer technique; protein-membrane interactions; amyloid; type 2 diabetes
(hIAPP; Figure 1), which is co-produced and cosecreted with insulin by the islet $\beta$-cells. hIAPP is overproduced in states of insulin resistance, a characteristic feature of type 2 diabetes, promoting its aggregation and fibril formation. Islet amyloidosis is thought to be an early factor responsible for $\beta$-cell failure (insulin insufficiency), which is strongly supported by the in vitro cytotoxic effect of hIAPP, as well as by the results of in vivo hIAPP transgenic mouse studies. ${ }^{1,2}$

It is clear from many studies that hIAPP interacts with membranes. ${ }^{3-15}$ Most likely the interaction results in membrane damage; however, details of the nature of this interaction are not known. Some studies suggest binding of hIAPP to the 


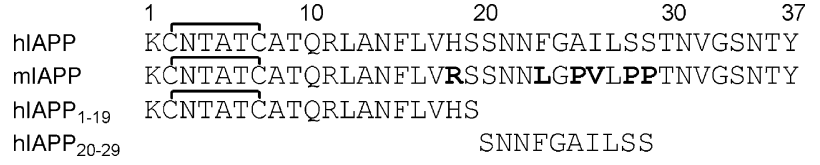

Figure 1. The amino acid sequences of hIAPP, mIAPP and the hIAPP fragments used in this study. The six residues in mIAPP that differ from the residues at corresponding positions in hIAPP are depicted in bold.

membrane, followed by loss of lipids from the membrane, ${ }^{14,15}$ whereas other studies suggest insertion of hIAPP into the membrane. ${ }^{12,13}$ It was also observed that phospholipids in a membrane are able to catalyse hIAPP amyloid formation, leaving the lipid bilayer intact. ${ }^{11}$ Besides the different types of hIAPP-membrane interactions that have been suggested, it is also unclear which of the hIAPP species, i.e. monomer, oligomer, protofibril or mature fibril, is mainly involved in membrane interactions. Several studies have indicated that hIAPP oligomers, and not the fibrils, are involved in the interaction with membranes. ${ }^{3-10}$ Such oligomers are thought to be intermediate species in the formation of hIAPP amyloid fibrils, and can consist of several monomers in a spherical arrangement. ${ }^{6}$ On the other hand, a recent study points to a possible role of hIAPP monomers in membrane interactions. ${ }^{14}$ In addition, it has been suggested that the process of hIAPP amyloid formation and not the presence of a particular hIAPP species is related to cytotoxicity. ${ }^{11,15}$ The results of the studies on hIAPP-membrane interactions yield a variety of sometimes contradicting ideas and hypotheses, indicating that the interaction between hIAPP and membranes is still far from being understood.

Here, we try to obtain insight into the interaction between hIAPP and membranes by focussing on the initial step of the interaction. The monolayer technique is used to differentiate between hIAPP binding on membranes and hIAPP insertion into membranes. This technique also allows us to study the nature and the molecular details of the initial step of the interaction between hIAPP and lipid membranes. The results indicate that the monomeric form of hIAPP has a pronounced ability to insert into phospholipid monolayers, whereas fibrillar hIAPP does not insert. Furthermore, it is observed that the insertion behaviour of freshly dissolved hIAPP is similar to the insertion behaviour of the non-cytotoxic and non-amyloidogenic mouse IAPP (mIAPP). Experiments with hIAPP fragments indicate that the conserved N-terminal part plays a major role in membrane insertion of both peptides.

\section{Results}

The initial step in the interaction between hIAPP and phospholipid membranes, leading to membrane damage, may consist of merely binding of
hIAPP to the surface of the membrane or it can involve actual insertion of hIAPP into the membrane. Here, we use monolayer experiments to study the nature of the initial step of the interaction between hIAPP and lipid membranes. Insertion of peptides into a monolayer of phospholipids at the air/water interface can be monitored as an increase in the surface pressure of the monolayer. Proteins that only interact with the lipid head groups without actually inserting between the lipid molecules, do not induce an increase in the surface pressure. $^{16}$ The monolayer experiments were performed with lipid monolayers composed of DOPC/ DOPS in a 7:3 molar ratio. This composition was chosen because these phospholipids represent the most abundant zwitterionic phospholipid species (PC) and the dominant negatively charged phospholipid species (PS) in eukaryotic cells. The 7:3 ratio was chosen because it resembles the ratio of zwitterionic lipids to negatively charged lipids of the membrane of pancreatic islet cells.

\section{Freshly dissolved hIAPP inserts into phospholipid monolayers}

Injection of a solution of freshly dissolved hIAPP in the aqueous sub-phase below a DOPC/DOPS (7:3) monolayer results in a fast increase in the surface pressure, followed by a plateau starting at approximately $5 \mathrm{~min}$ after addition of the peptide (Figure 2(a)). However, when hIAPP is first incubated in buffer for $21 \mathrm{~h}$, allowing amyloid fibrils to form, its ability to insert into the lipid monolayer disappears completely (Figure 2(a)). In fact, the ability of hIAPP to induce an increase in surface pressure disappears already after $4 \mathrm{~h}$ of incubation in buffer (Figure 2(b)). This incubation time is consistent with the time required for the formation of hIAPP fibrils, as observed by electron microscopy (results not shown).

To check whether the insertion behaviour is related to the process of fibril formation, we performed similar experiments with the nonamyloidogenic and non-cytotoxic mouse IAPP (mIAPP). ${ }^{18}$ The amino acid sequence of mIAPP is similar to that of hIAPP, but contains proline residues at positions 25,28 and 29 that prevent fibril formation (Figure 1). ${ }^{19}$ In addition, there are no indications that mIAPP can oligomerize, hence we assume in this study that mIAPP remains a monomer. Mouse IAPP is therefore a suitable and biologically relevant reference peptide in the study of the interaction of hIAPP with membranes.

Freshly dissolved mIAPP inserts into phospholipid monolayers and gives rise to an increase in the surface pressure at approximately the same rate as hIAPP (Figure 2(a)). The increase in surface pressure induced by insertion of mIAPP is $5 \mathrm{mN} / \mathrm{m}$, compared to an increase of $7 \mathrm{mN} / \mathrm{m}$ for freshly dissolved hIAPP (Figure 2(a)). Incubation of mIAPP in buffer for $21 \mathrm{~h}$ does not reduce its capacity to insert. Since mIAPP does not form fibrils or oligomers, it is likely that the monomeric form of 

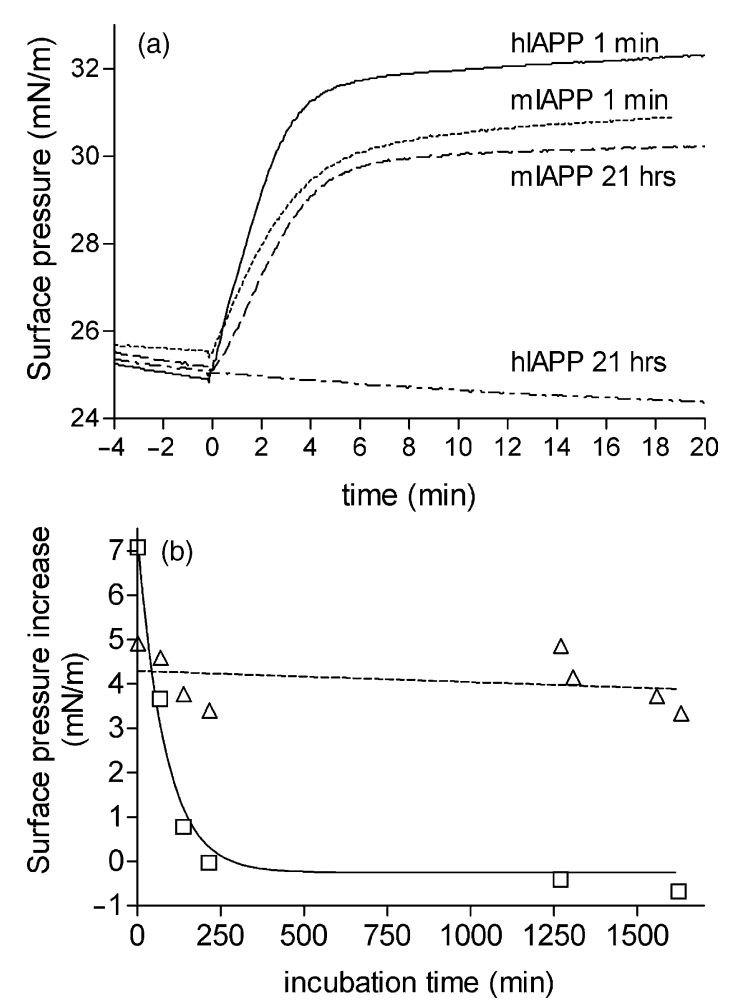

Figure 2. Effect of incubation time of hIAPP and mIAPP in buffer on their insertion in a monolayer of DOPC/ DOPS (7:3). (a) Surface pressure profile after injecting a sample of hIAPP incubated in buffer for 1 min (continuous line) or for $21 \mathrm{~h}$ (dash-dot line), and a sample of mIAPP incubated in buffer for $1 \mathrm{~min}$ (dotted line) or for $21 \mathrm{~h}$ (broken line).(b) Effect of incubation time of hIAPP (squares) and mIAPP (triangles) in buffer on the surface pressure increase at initial surface pressures of $25 \mathrm{mN} / \mathrm{m}$. The peptide was injected into the stirred sub-phase at $t=$ $0 \mathrm{~min}$. The final peptide concentration was $0.25 \mu \mathrm{M}$. The buffer consists of $50 \mathrm{mM}$ Hepes ( $\mathrm{pH} 7.0$ ).

mIAPP is responsible for its insertion behaviour. Considering the high level of sequence similarity between mIAPP and hIAPP, and taking into account that the fibrillar form of hIAPP does not insert, we propose that also in the case of hIAPP, its monomeric form is responsible for the observed insertion into lipid monolayers.

\section{High insertion ability could indicate insertion of IAPP in membranes in vivo}

The packing density of lipids in biological membranes corresponds to surface pressures between $31 \mathrm{mN} / \mathrm{m}$ and $35 \mathrm{mN} / \mathrm{m}^{20}$ To test the ability of hIAPP to insert into membranes in vivo, we determined the maximal initial surface pressure at which hIAPP can still cause an increase in surface pressure, a property also known as the "limiting surface pressure". The results show that both freshly dissolved hIAPP and mIAPP insert in monolayers composed of DOPC/DOPS (7:3) with a similar dependence on the initial surface pressure

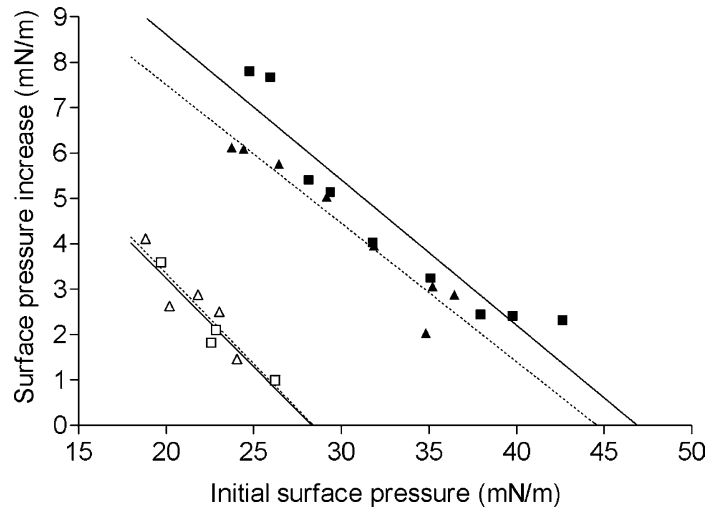

Figure 3. Surface pressure increase induced by the interaction of freshly dissolved hIAPP and mIAPP with lipid monolayers of different composition as a function of the initial surface pressure. The interaction of freshly dissolved hIAPP with monolayers composed of DOPC (open squares) and DOPC/DOPS (filled squares), and the interaction of mIAPP with monolayers composed of DOPC (open triangles) and DOPC/DOPS (filled triangles) is shown. The straight lines were obtained by linear regression. Other experimental conditions are the same as described in the legend to Figure 2.

(Figure 3). The surface pressure increase for both peptides has a nearly linear dependence on the initial surface pressure, a relation that is common for many peptides. The limiting surface pressure is high for both peptides and appears to be slightly lower for mIAPP $(45 \mathrm{mN} / \mathrm{m})$ compared to hIAPP $(47 \mathrm{mN} / \mathrm{m})$. In both cases, the limiting surface pressure is significantly higher than the surface pressures that correspond to the packing density of lipids in biological membranes. The results thus indicate that both mIAPP and freshly dissolved hIAPP are likely able to insert into biological membranes in vivo.

\section{Electrostatic interactions are important for insertion}

The similarities in both the kinetics of insertion and the dependence of the insertion on the initial surface pressure between mIAPP and freshly dissolved hIAPP are most likely due to the similarities in the primary structure of the two peptides. One such similarity is the presence of positively charged residues in both peptides, which might be responsible for electrostatic interactions with phospholipids. To test the importance of electrostatic interactions, we compared the insertion of both peptides in monolayers containing negatively charged lipids, with the insertion in monolayers containing only zwitterionic lipids.

The insertion behaviour in monolayers composed of only zwitterionic lipids (DOPC) is nearly identical for both peptides, but both the surface pressure increase as well as the limiting surface pressure are much less compared to insertion in monolayers containing negatively charged lipids (Figure 3). These results indicate that electrostatic 
interactions between the negatively charged head group of DOPS and the positively charged residues of the peptides are a dominant factor in the insertion of hIAPP and mIAPP into phospholipid monolayers. This is in contrast to the results of a recent study, in which it was observed that the presence of negatively charged lipids had no effect on the insertion of hIAPP at surface pressures below $20 \mathrm{mN} / \mathrm{m} \cdot{ }^{13}$ A likely explanation is the high surface activity of the peptide itself, which may dominate any other effect at this low initial surface pressure. In our study, initial surface pressures lower than $20 \mathrm{mN} / \mathrm{m}$ were not used, since hIAPP and mIAPP gave rise to surface pressures of $19 \mathrm{mN} / \mathrm{m}$ in the absence of a lipid monolayer (results not shown).

The strong insertion in monolayers containing DOPS was not due to a specific interaction of hIAPP or mIAPP with DOPS, since similar limiting surface pressures were found in monolayers containing DOPG, a lipid that is also negatively charged, but has a different head group (results not shown). The importance of electrostatic interactions was confirmed by the observation that increased ionic strength, up to $0.5 \mathrm{M} \mathrm{NaCl}$, significantly lowered the surface pressure increase induced by both hIAPP and mIAPP in DOPC/DOPS monolayers, most likely as a result of charge shielding by the salt (results not shown).

\section{The N-terminal part of hIAPP is responsible for insertion in membranes}

The similar insertion behaviour of freshly dissolved hIAPP and mIAPP (Figure 3) and the location of positively charged residues indicate that the N-terminal part of IAPP might be important for insertion. To test this, we studied the insertion behaviour of two hIAPP fragments. The first fragment is a peptide composed of the first 19 amino acid residues of hIAPP (hIAPP $\left.{ }_{1-19}\right)$, which is nearly identical with the N-terminal part of mIAPP. The second fragment, hIAPP $_{20-29}$, is composed of amino acid residues 20 to 29 of hIAPP, and is able to form amyloid fibrils in vitro but lacks the N-terminal part. ${ }^{19,21}$ The sequence of the latter peptide differs considerably from the corresponding region of mIAPP, with only $50 \%$ identity (Figure 1).

The insertion of hIAPP ${ }_{1-19}$ is clearly enhanced by the presence of negatively charged lipids, as seen with full-length hIAPP, but the higher limiting surface pressure of hIAPP $_{1-19}$ indicates that it inserts even better than full-length hIAPP (Figure 4). In contrast, the insertion of hIAPP $20-29$ occurs at much lower surface pressures compared to hIAPP $_{1-19}$, and is almost the same in the absence and in the presence of negatively charged lipids. These results show that the presence of the amyloidogenic 20-29 region is not a requirement for interaction with lipids. In fact, it can be concluded that the N-terminal part of hIAPP, which is nearly identical with the N-terminal part

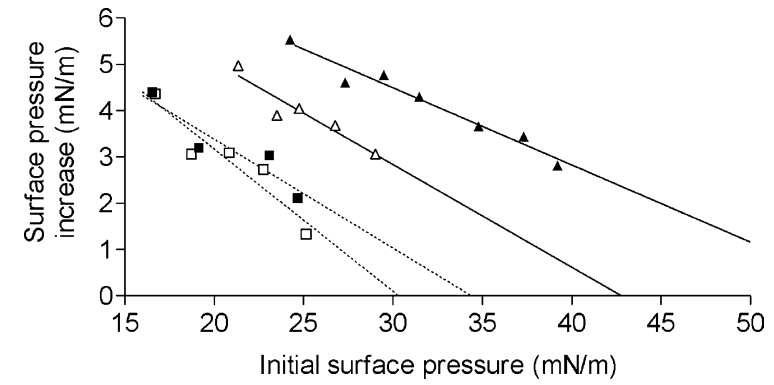

Figure 4. Surface pressure increase induced by the interaction of freshly dissolved hIAPP fragments with monolayers of different composition as a function of the initial surface pressure. The interaction of freshly dissolved IAPP ${ }_{1-19}$ with monolayers composed of DOPC (open triangles) and DOPC/DOPS (filled triangles) and the interaction of IAPP $_{20-29}$ with monolayers composed of DOPC (open squares) and DOPC/DOPS (filled squares) is shown. The straight lines were obtained by linear regression. Other experimental conditions are the same as described in the legend to Figure 2.

of the non-amyloidogenic mIAPP, is largely responsible for insertion into phospholipid monolayers.

\section{Discussion}

In this study, we have performed monolayer experiments to obtain insight into the nature and the molecular details of the initial step of hIAPPmembrane interactions. It is shown that freshly dissolved hIAPP has a pronounced ability to insert into lipid monolayers, whereas fibrillar hIAPP does not insert. The insertion behaviour of the nonamyloidogenic mIAPP is similar to that of freshly dissolved hIAPP. Both mIAPP and freshly dissolved hIAPP are able to insert into monolayers with lipid packing densities that exceed lipid packing densities found in biological membranes, ${ }^{20}$ suggesting that both peptides might insert in biological membranes in vivo. Monolayer experiments with hIAPP fragments show that the N-terminal part of hIAPP, and not the amyloidogenic 20-29 region, plays a major role in insertion of the peptide. These observations give important insights into the initial step in hIAPP-membrane interactions, as discussed below.

\section{The ability to form fibrils is not required for insertion}

An important finding of this study is that freshly dissolved hIAPP and mIAPP insert into a lipid monolayer, and do not just adsorb to the surface of the membrane, as proposed recently. ${ }^{11}$ It is shown here that the N-terminal part of the peptide, which is highly conserved among different species, ${ }^{22}$ plays a major role in the insertion of the peptide into lipid monolayers. Our observation that hIAPP $_{1-19}$ displays very strong insertion, combined with the fact 
that the N-terminal parts of hIAPP and mIAPP are nearly identical, suggests that this part of the peptide is important for the observed insertion behaviour. Most likely, the insertion is governed by electrostatic interactions between the positively charged N-terminal residues and the negatively charged lipid head groups. This is consistent with our previous observation that indicated that the presence of negatively charged lipids accelerates hIAPP-induced leakage of giant unilamellar lipid vesicles. $^{15}$

In addition to the observations that mIAPP (or the identical rat IAPP) is not cytotoxic and does not form amyloid, ${ }^{18}$ it has been shown that mIAPP does not permeabilize lipid vesicles ${ }^{7,14,15}$ and that it does not increase membrane conductance. ${ }^{3,4}$ Neutron diffraction measurements seem to indicate the absence of insertion of mIAPP into the hydrophobic chain region of membrane lipids. ${ }^{12}$ However, in recent reports it was shown that there is an interaction between mIAPP and lipid bilayers. ${ }^{11,14,23}$ Both freshly dissolved hIAPP and mIAPP have been shown to undergo a transformation from random structure to $\alpha$-helical structure induced by lipid vesicles. ${ }^{23}$ In addition, it was found that mIAPP is able to induce defects in lipid bilayers as observed by AFM. ${ }^{14}$ Our results suggest that these observations can be explained by insertion of mIAPP into lipid bilayers.

\section{Insertion of hIAPP monomers is the first step in hIAPP-membrane interactions}

The similarity between the insertion behaviour of mIAPP and freshly dissolved hIAPP, and the observation that the aggregated form of hIAPP has lost the ability to insert, combined with the importance of the $\mathrm{N}$-terminal part for insertion of the peptide, are strong arguments that point to a main role for monomers in hIAPP-induced increase in surface pressure of lipid monolayers. Unfortunately, direct evidence for the conclusion that monomers are the responsible species is very hard to obtain. We propose a model in which insertion of monomeric hIAPP into lipid bilayers precedes membrane-associated formation of hIAPP amyloid fibrils, as is shown schematically in Figure 5. Our results indicate that the amyloidogenic 20-29 region remains available for fibril formation when hIAPP inserts via its $\mathrm{N}$-terminal part, as has also been suggested recently. ${ }^{24}$ In addition, a model of hIAPP fibrils shows that the $\mathrm{N}$-terminal residues are not part of the fibril core. ${ }^{25}$ We therefore suggest that insertion of hIAPP monomers in the membrane and subsequent participation of the inserted hIAPP monomers in oligomer and fibril formation at the membrane are important steps in the interaction of hIAPP with membranes (Figure 5).

Previously, we hypothesized that membrane damage is induced by the process of hIAPP amyloid formation and is accompanied by uptake of lipids from the membrane in forming hIAPP amyloid. ${ }^{15}$

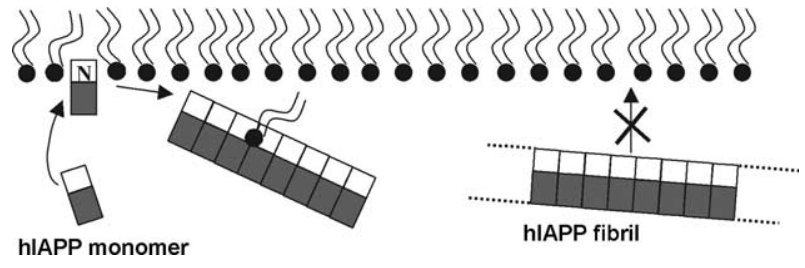

Figure 5. Proposed model for the interaction of hIAPP with a phospholipid monolayer. Insertion of hIAPP monomers into the lipid monolayer precedes hIAPP fibril formation. Fibril formation occurs near the bilayer, with participation of inserted monomers, and is accompanied by the occasional uptake of lipid molecules into the forming hIAPP amyloid. Mature fibrils do not insert into the phospholipid monolayer.

The strong indication that hIAPP monomers insert into lipid membranes is compatible with this hypothesis. When IAPP monomers that are inserted in lipid membranes participate in fibril formation, as we expect, it would be plausible that lipid molecules are taken up in the forming hIAPP amyloid, as we have indeed observed. ${ }^{15}$ The uptake of lipids in forming amyloid has been observed for other amyloidogenic proteins as well, which indicates that lipid uptake is a common property of amyloid-forming proteins and peptides in the presence of lipid membranes. ${ }^{26}$ Indeed, analysis of amyloid tissue from patients shows that in vivo formed amyloid fibrils contain up to $10 \%$ lipids. $^{27}$ These notions, combined with the results obtained here, indicate that insertion of monomeric hIAPP in the membrane, followed by hIAPP-induced membrane damage by the uptake of lipids in forming amyloid, is a plausible mechanism to explain the cytotoxic effect of hIAPP in type 2 diabetes, contributing to $\beta$-cell failure.

\section{Materials and Methods}

\section{Materials}

hIAPP was obtained from Bachem AG (Bubendorf, Switzerland) and mIAPP was obtained from Pepscan (Lelystad, The Netherlands). The calculated mass of the peptides was confirmed by mass spectrometry. 1,2-Dioleoyl-sn-glycero-3-phosphocholine (DOPC), 1,2dioleoyl-sn-glycero-3-phospho-L-serine (DOPS) and 1,2dioleoyl-sn-glycero-3-phospho-rac-(1-glycerol) (DOPG) were obtained from Avanti Polar Lipids (Alabaster, AL). The peptide fragments hIAPP $_{1-19}$ and hIAPP $20-29$ were synthesized as described. ${ }^{28}$ Linear hIAPP ${ }_{1-19}$ was dissolved in aqueous dimethyl sulfoxide (DMSO) and oxidized to the corresponding disulfide. ${ }^{29}$ The peptides were purified by HPLC and characterized by MALDITOF mass spectrometry.

\section{Monolayer experiments}

Peptide-induced changes in the surface pressure of a monomolecular layer of phospholipids at constant 
surface area were measured with the Wilhelmy plate method. ${ }^{30}$ Surface pressures were measured at $22( \pm 2){ }^{\circ} \mathrm{C}$ using a Cahn 2000 microbalance. A Teflon trough was filled with $6.0 \mathrm{ml}$ of freshly filtered $50 \mathrm{mM}$ Hepes buffer (pH 7.0). The sub-phase was continuously stirred with a magnetic bar during the measurements. Lipid monolayers were spread from a $1 \mathrm{mM}$ lipid solution in chloroform at the air/buffer interface to give initial surface pressures between $20 \mathrm{mN} / \mathrm{m}$ and $43 \mathrm{mN} / \mathrm{m}$. The lipid monolayer was allowed to stabilize for a few minutes before $6 \mu \mathrm{l}$ of a $250 \mu \mathrm{M}$ stock solution of the peptide in DMSO was injected in the sub-phase without disturbing the lipid monolayer, resulting in a final peptide concentration of $0.25 \mu \mathrm{M}$. Higher peptide concentrations did not significantly increase the surface pressure. The peptide concentration of the stock solution was determined by weighing an amount of the freezedried peptide powder, which was subsequently dissolved in an appropriate volume of DMSO. The peptide stock solutions were used within one day, and were prepared at least $1 \mathrm{~h}$ before the start of the experiment, to allow for complete dissolution of the peptide. DMSO alone, at the concentrations used here $(0.1 \%(\mathrm{v} / \mathrm{v}))$, did not affect the surface pressure of the monolayers.

\section{Acknowledgements}

We thank Jacob Gubbens and Berthil Prinsen for performing mass spectrometry analysis. This research was financially supported by the Dutch Diabetes Research Foundation (grant no. 2002.00.019).

\section{References}

1. Höppener, J. W. M., Ahrén, B. \& Lips, C. J. M. (2000). Islet amyloid and type 2 diabetes mellitus. New Engl. J. Med. 343, 411-419.

2. Hull, R. L., Westermark, G. T., Westermark, P. \& Kahn, S. E. (2004). Islet amyloid: a critical entity in the pathogenesis of type 2 diabetes. J. Clin. Endocrinol. Metab. 89, 3629-3643.

3. Mirzabekov, T. A., Lin, M. C. \& Kagan, B. L. (1996). Pore formation by the cytotoxic islet amyloid peptide amylin. J. Biol. Chem. 271, 1988-1992.

4. Janson, J., Ashley, R. H., Harrison, D., McIntyre, S. \& Butler, P. C. (1999). The mechanism of islet amyloid polypeptide toxicity is membrane disruption by intermediate-sized toxic amyloid particles. Diabetes, 48, 491-498.

5. Kayed, R., Head, E., Thompson, J. L., McIntire, T. M., Milton, S. C., Cotman, C. W. \& Glabe, C. G. (2003). Common structure of soluble amyloid oligomers implies common mechanism of pathogenesis. Science, 300, 486-489.

6. Porat, Y., Kolusheva, S., Jelinek, R. \& Gazit, E. (2003). The human islet amyloid polypeptide forms transient membrane-active prefibrillar assemblies. Biochemistry, 42, 10971-10977.

7. Anguiano, M., Nowak, R. J. \& Lansbury, P. T., Jr (2002). Protofibrillar islet amyloid polypeptide permeabilizes synthetic vesicles by a pore-like mechanism that may be relevant to type II diabetes. Biochemistry, 41, 11338-11343.
8. Demuro, A., Mina, E., Kayed, R., Milton, S. C., Parker, I. \& Glabe, C. G. (2005). Calcium dysregulation and membrane disruption as a ubiquitous neurotoxic mechanism of soluble amyloid oligomers. J. Biol. Chem. 280, 17294-17300.

9. Kayed, R., Sokolov, Y., Edmonds, B., McIntire, T. M., Milton, S. C., Hall, J. E. \& Glabe, C. G. (2004). Permeabilization of lipid bilayers is a common conformation-dependent activity of soluble amyloid oligomers in protein misfolding diseases. J. Biol. Chem. 279, 46363-46366.

10. Quist, A., Doudevski, I., Lin, H., Azimova, R., $\mathrm{Ng}$, D., Frangione, B. et al. (2005). Amyloid ion channels: a common structural link for proteinmisfolding disease. Proc. Natl Acad. Sci. USA, 102, 10427-10432.

11. Knight, J. D. \& Miranker, A. D. (2004). Phospholipid catalysis of diabetic amyloid assembly. J. Mol. Biol. 341, 1175-1187.

12. Balali-Mood, K., Ashley, R. H., Hauß, T. \& Bradshaw, J. P. (2005). Neutron diffraction reveals sequence-specific membrane insertion of pre-fibrillar islet amyloid polypeptide and inhibition by rifampicin. FEBS Letters, 579, 1143-1148.

13. Harroun, T. A., Bradshaw, J. P. \& Ashley, R. H. (2001). Inhibitors can arrest the membrane activity of human islet amyloid polypeptide independently of amyloid formation. FEBS Letters, 507, 200-204.

14. Green, J. D., Kreplak, L., Goldsbury, C., Li Blatter, X., Stolz, M., Cooper, G. S. et al. (2004). Atomic force microscopy reveals defects within mica supported lipid bilayers induced by the amyloidogenic human amylin peptide. J. Mol. Biol. 342, 877-887.

15. Sparr, E., Engel, M. F. M., Sakharov, D. V., Sprong, M., Jacobs, J., de Kruijff, B. et al. (2004). Islet amyloid polypeptide-induced membrane leakage involves uptake of lipids by forming amyloid fibers. FEBS Letters, 577, 117-120.

16. Demel, R. A., London, Y., Geurts van Kessel, W. S., Vossenberg, F. G. \& van Deenen, L. L. (1973). The specific interaction of myelin basic protein with lipids at the air-water interface. Biochim. Biophys. Acta, 311, 507-519.

17. Rustenbeck, I., Matthies, A. \& Lenzen, S. (1994). Lipid composition of glucose-stimulated pancreatic islets and insulin-secreting tumor cells. Lipids, 29, 685-692.

18. Lorenzo, A., Razzaboni, B., Weir, G. C. \& Yankner, B. A. (1994). Pancreatic islet cell toxicity of amylin associated with type-2 diabetes mellitus. Nature, 368, 756-760.

19. Westermark, P., Engström, U., Johnson, K. H., Westermark, G. T. \& Betsholtz, C. (1990). Islet amyloid polypeptide: pinpointing amino acid residues linked to amyloid fibril formation. Proc. Natl Acad. Sci. USA, 87, 5036-5040.

20. Demel, R. A., Geurts van Kessel, W. S., Zwaal, R. F., Roelofsen, B. \& van Deenen, L. L. (1975). Relation between various phospholipase actions on human red cell membranes and the interfacial phospholipid pressure in monolayers. Biochim. Biophys. Acta, 406, 97-107.

21. Goldsbury, C., Goldie, K., Pellaud, J., Seelig, J., Frey, P., Müller, S. A. et al. (2000). Amyloid fibril formation from full-length and fragments of amylin. J. Struct. Biol. 130, 352-362. 
22. Nishi, M., Chan, S. J., Nagamatsu, S., Bell, G. I. \& Steiner, D. F. (1989). Conservation of the sequence of islet amyloid polypeptide in five mammals is consistent with its putative role as an islet hormone. Proc. Natl Acad. Sci. USA, 86, 5738-5742.

23. Jayasinghe, S. A. \& Langen, R. (2005). Lipid membranes modulate the structure of islet amyloid polypeptide. Biochemistry, 44, 12113-12119.

24. Kurganov, B., Doh, M. \& Arispe, N. (2004). Aggregation of liposomes induced by the toxic peptides Alzheimer's Aßs, human amylin and prion (106-126): facilitation by membrane-bound $\mathrm{G}_{\mathrm{M} 1}$ ganglioside. Peptides, 25, 217-232.

25. Kajava, A. V., Aebi, U. \& Steven, A. C. (2005). The parallel superpleated beta-structure as a model for amyloid fibrils of human amylin. J. Mol. Biol. 348, 247-252.

26. Zhao, H., Tuominen, E. K. J. \& Kinnunen, P. K. J. (2004). Formation of amyloid fibers triggered by phosphatidylserine-containing membranes. Biochemistry, 43, 10302-10307.

27. Gellermann, G. P., Appel, T. R., Tannert, A., Radestock, A., Hortschansky, P., Schroeckh, V. et al. (2005). Raft lipids as common components of human extracellular amyloid fibrils. Proc. Natl Acad. Sci. USA, 102, 6297-6302.

28. Rijkers, D. T. S., Höppener, J. W. M., Posthuma, G., Lips, C. J. M. \& Liskamp, R. M. J. (2002). Inhibition of amyloid fibril formation of human amylin by $\mathrm{N}$-alkylated amino acid and alpha-hydroxy acid residue containing peptides. Chem. Eur. J. 8, 4285-4291.

29. Tam, J. P., Wu, C. R., Liu, W. \& Zhang, J. W. (1991). Disulfide bond formation in peptides by dimethylsulfoxide-scope and applications. J. Am. Chem. Soc. 113, 6657-6662.

30. Demel, R. A. (1994). Monomolecular layers in the study of biomembranes. Subcell. Biochem. 23, 83-120.

Edited by G. von Heijne

(Received 4 October 2005; received in revised form 5 December 2005; accepted 6 December 2005) Available online 21 December 2005 